\title{
AN AUDIT OF THE MANAGEMENT OF IRON DEFICIENCY ANAEMIA
}

\author{
Usha Thilliambalam, Senior House Officer; \\ CM Brown, Consultant Physician and Gastroenterologist \\ Royal Lancaster Infirmary
}

\section{INTRODUCTION}

\section{Type of anaemia}

Iron deficiency anaemia (IDA) is a common clinical problem in primary care and in many different hospital departments. It is usually recognised by a microcytic hypochromic picture on a blood film or full blood count, a mean red cell volume $(\mathrm{MCV})$ of less than $75 \mathrm{fl}$ and reduced mean corpuscular haemoglobin $(\mathrm{MCH})$ and mean corpuscular haemoglobin concentration (MCHC). Thalassaemia minor and (occasionally) an anaemia of chronic disorder may account for a microcytic anaemia, but not commonly in Morecambe Bay.

\section{Causes of IDA}

The most common cause of IDA is menstrual loss in young and middle-aged women. In these healthy subjects invasive gastrointestinal investigations are usually not required. The cause of IDA, however, should be investigated in all adult males and in females over the age of 50 and is usually caused by occult bleeding from the gastrointestinal tract. Obvious symptoms such as haemoptysis or frank haematuria will direct investigations appropriately.

The cause of IDA can be determined in $90-95 \%$ of fully investigated subjects, which therefore leaves a small fraction of patients in whom the cause is obscure. The most important conditions presenting with IDA include gastric and duodenal ulcer (Figure 1), coeliac disease and gastric and colorectal cancer (Figure 2). It is unwise falsely to attribute mild oesophagitis, gastritis and duodenitis and mild diverticular disease as the cause of IDA without suspecting and excluding more serious disease. Furthermore, the authors do not believe that a hiatal hernia per se causes IDA, which is a historical "old wives' tale" still noted in older textbooks.

\section{Investigation of IDA}

Upper gastrointestinal symptoms such as dyspepsia together with IDA should prompt a gastroscopy ${ }^{(1)}$. In the absence of a major endoscopic finding such as cancer or peptic ulcer, the endoscopist should consider obtaining biopsies from the distal or lower duodenum to look for coeliac disease, a treatable cause of anaemia.

IDA and colorectal symptoms such as rectal bleeding, constipation, diarrhoea, mid or lower abdominal pain should prompt investigation of the colon. The choice of investigation lies between a rigid or flexible sigmoidoscopy plus a barium enema, or a colonoscopy ${ }^{(1)}$. The advantages of colonoscopy include the ability to biopsy lesions and perform therapeutic manoeuvres such as polypectomy. Limitations compared with barium enema include its technical difficulties in some patients, need for sedation and increased risks of perforation (approximately 1 in 1000), particularly in frail elderly patients. The choice of investigation lies with the clinician, but the right side of the colon and caecum must be adequately visualised

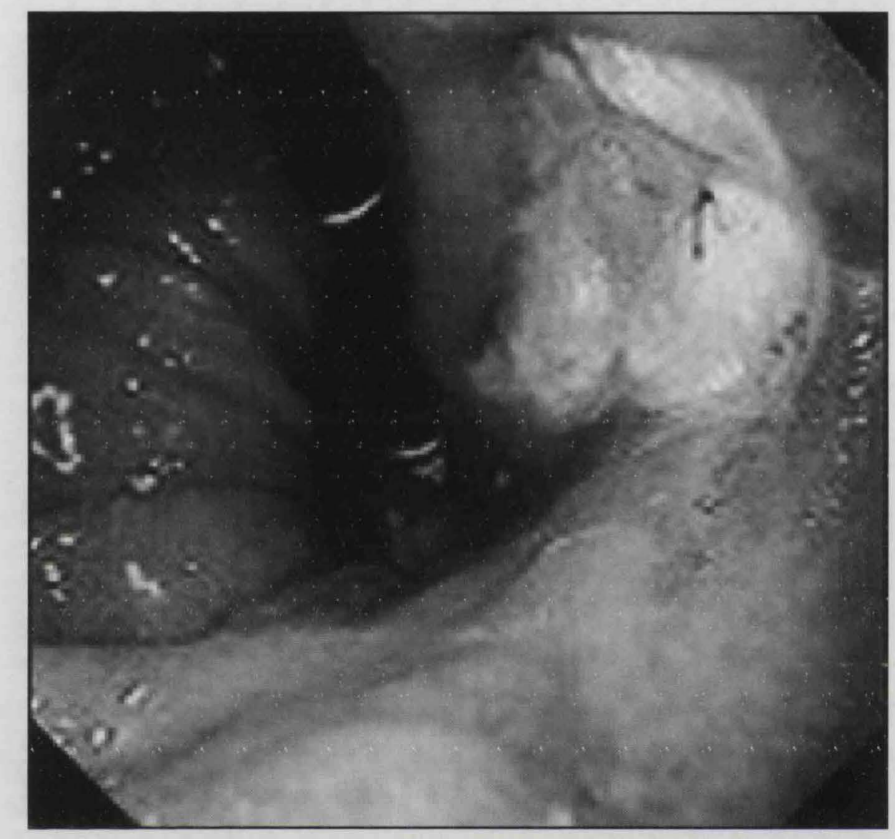

Figure 1 Endoscopy revealing a gastric ulcer

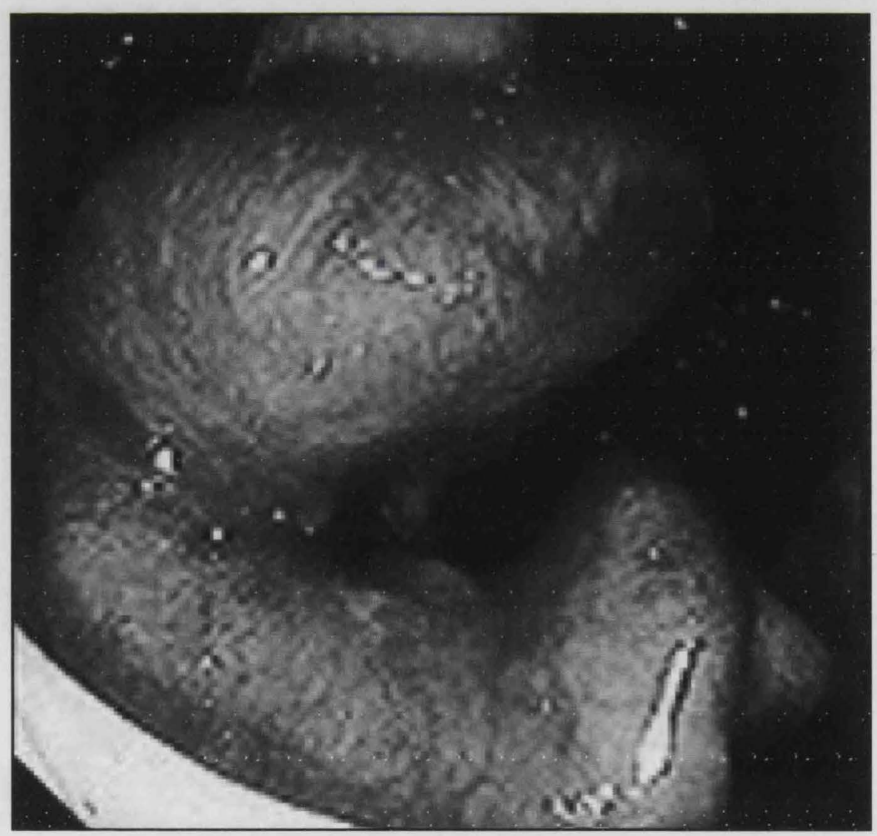

Figure 2 Colonoscopy revealing a colonic carcinoma

and in some cases both techniques may be complementary. Relative waiting times for barium enemas and colonoscopy also influence clinicians' choice.

Where there are no symptoms to indicate an upper or a lower gastrointestinal cause for IDA, it is sometimes helpful to perform a combined gastroscopy and colonoscopy for the patient as a day case. 
Faecal occult blood (FOB) testing has little or no role in the routine investigation of IDA in the authors' view, since the GI tract should be investigated regardless of FOB results except in women under 50 years. There are only two situations in which the authors pursue FOB testing: firstly, when a patient reports frequent dark stools but the doctor is suspicious that the patient is not bleeding (not in the setting of anaemia); secondly, in younger women with IDA following a normal gastroscopy and distal duodenal biopsy without colonic symptoms, in whom negative FOBs suggest a colonoscopy is not required. FOB testing is being systematically researched as a population screening tool for asymptomatic colorectal cancer and may lead to reducing colorectal cancer-associated mortality by about one third. A major change in National Health policy is required to screen for colon cancer with significant enhancement of diagnostic facilities (endoscopy and radiology) and such a policy is about to be piloted in England and Scotland.

\section{AUDITING THE MANAGEMENT OF IDA}

There are several recent studies which have analysed the outcomes of patients with IDA and also the degree to which these patients have been under-investigated ${ }^{(2,3)}$. A recurring theme is that the most frequently missed serious lesion is a colon cancer. It is important to judge the clinical appropriateness of investigating IDA in individual cases and to determine the extent of investigation. For instance, it may be appropriate to pursue a treatable benign peptic ulcer in a patient with a moderately severe congestive heart failure or dementia, but inappropriate to pursue the possibility of a small asymptomatic colon cancer when a laparotomy would be hazardous.

There was a perception that the management of IDA in Lancaster and Kendal was no better and no worse to that seen in nationally published data. We therefore decided to audit the management and outcomes in patients with IDA within the medical department in a retrospective cohort of patients using pre-defined "gold standard criteria." We were specifically looking for the degree of under-investigation in these patients and the numbers of important "missed" diagnoses. Any lessons learned and the acceptance of guidelines for management of IDA may be applicable to other clinicians dealing with these patients, including haematologists, general surgeons and general practitioners.

\section{AUDIT METHODOLOGY}

A search was made for incident cases of IDA (new cases) being managed by consultant physicians within the department of medicine between January 1994 and December 1995. The haematology computer database was analysed for males with a haemoglobin less than $12.5 \mathrm{~g} / \mathrm{dl}$ (MCV $75 \mathrm{fl}$ or less) and females with a haemoglobin less than $11.5 \mathrm{~g} / \mathrm{dl}$ (MCV 75 or less). Exclusions included prevalent cases and those managed within other hospital departments.

Ninety-two cases of IDA were identified from within the medical unit, of which 77 casenotes were retrieved for analysis against a pre-determined proforma. Thirteen cases were then excluded from further scrutiny because of a likely nongastrointestinal cause for bleeding, or previously known anaemia, leaving 64 cases for full analysis (Figure 3 ).

\section{Audit results}

There were 35 males with mean haemoglobin $8.8 \mathrm{~g} / \mathrm{dl}$ (range 5.1 to 12.2 ) and 29 females with mean haemoglobin $9.1 \mathrm{~g} / \mathrm{dl}$

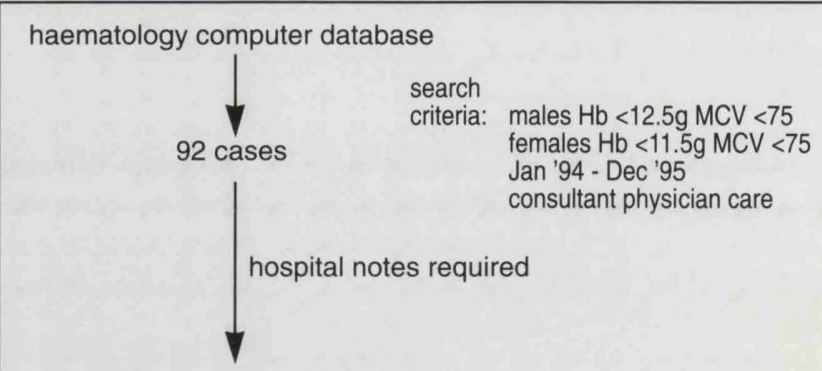

77 hospital casenotes retrieved

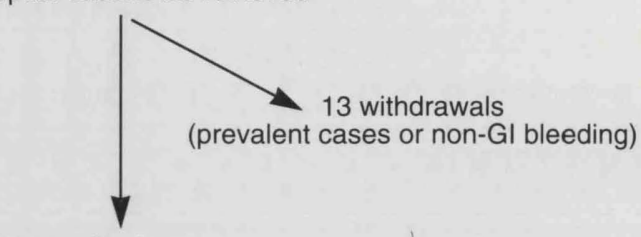

64 audited cases

Audit case selection

Figure 3 Selection of cases with iron deficiency anaemia for audit

(range 4.3 to 11.4). The two groups had similar MCVs (66 vs 71 respectively). The majority of these patients $(67 \%)$ had no gastrointestinal symptoms, whilst $22 \%$ had symptoms relating to the upper gut and $6 \%$ had colonic symptoms.

Only 13 of 64 patients had their iron deficiency confirmed with a low serum ferritin, although this may not always be necessary. There were inadequacies in the treatment of IDA with low rates of provision of oral iron tablets $(42 \%)$, probably excessive treatment with autologous blood transfusion (20\%) and inadequate assessment of the effectiveness of therapy with repeat haemoglobin checks in only 20 of 64 cases (Figure 4).

\begin{tabular}{|l|c|c|}
\hline Treatment & Number & Percentage \\
\hline Blood transfusion & 13 & 20 \\
\hline Iron tablets & 27 & 42 \\
\hline $\begin{array}{l}\text { Repeat haemoglobin after } \\
\text { treatment }\end{array}$ & 20 & 31 \\
\hline
\end{tabular}

Figure 4 Treatment of iron deficiency anaemia $(n=64)$

Thirty-three patients of 64 were investigated for IDA whilst almost $50 \%$ were not investigated at all (Figure 5). Six of the 31 patients not investigated for a cause of IDA were deemed medically unfit - one patient with dementia and those with lymphomas and cancer. The decisions not to investigate these cases were appropriate. A treatable cause such as coeliac disease could have been missed in the patient with lifelong anaemia, whilst no reasons were stated for 13 patients.

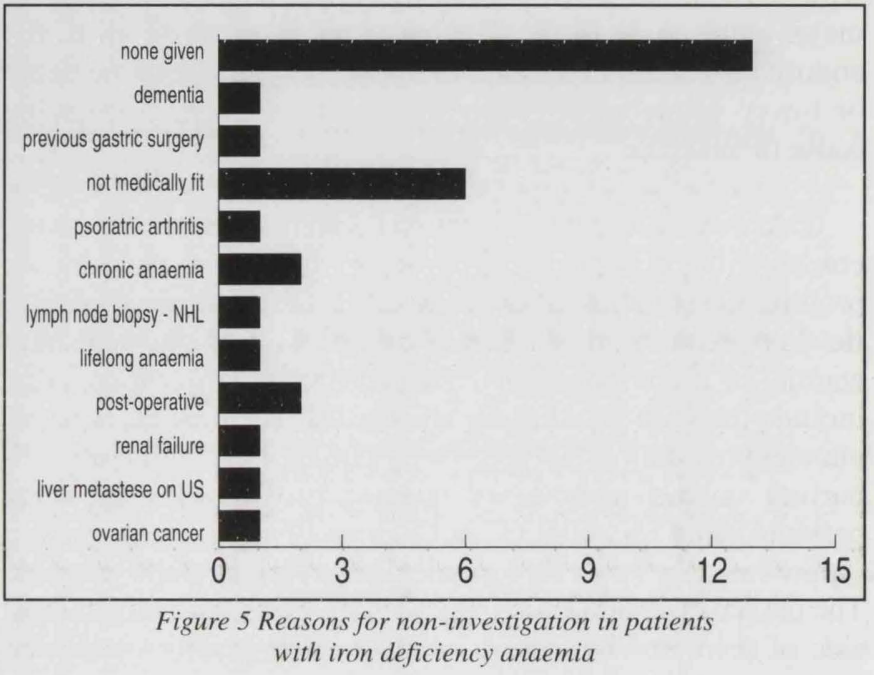


Unfortunately, in common with other clinical audits, it is only possible to audit documented clinical information.

In the 33 investigated patients, the type and number of tests are documented in Figure 6 and the range of diagnoses is noted in Figure 7. It is noteworthy that major findings of six gastrix ulcers, one duodenal ulcer and one colonic cancer were present in ten patients. There were a few cases of Crohn's disease, diverticular disease and presumed NSAID enteropathy. Oesophagitis and hiatal hernia were found in 12 patients.

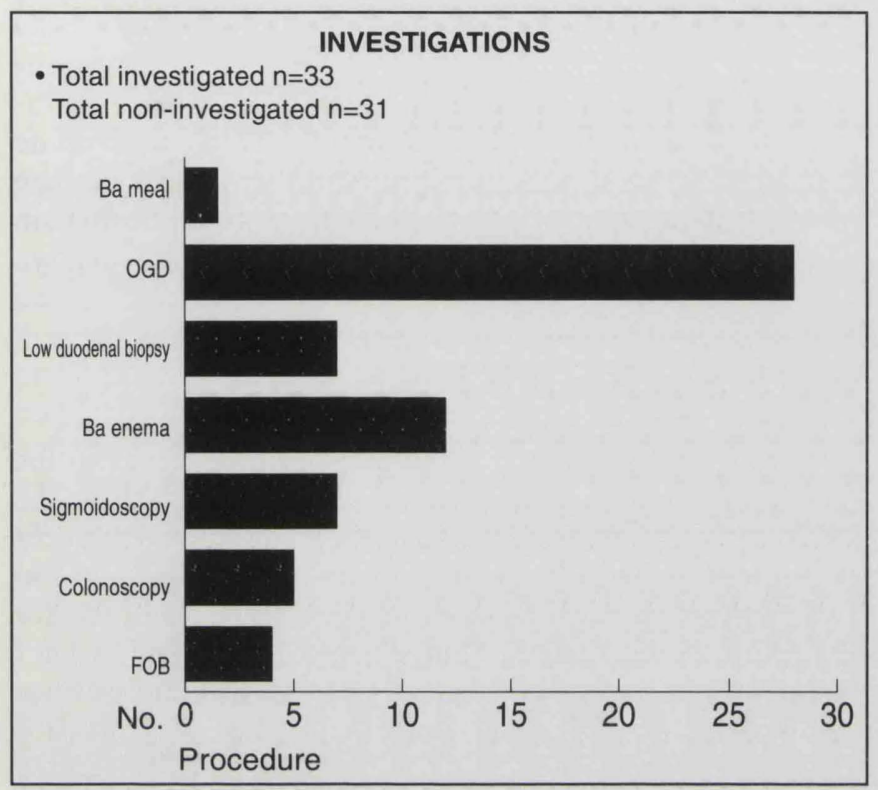

Figure 6 Investigations performed in patients with iron deficiency anaemia

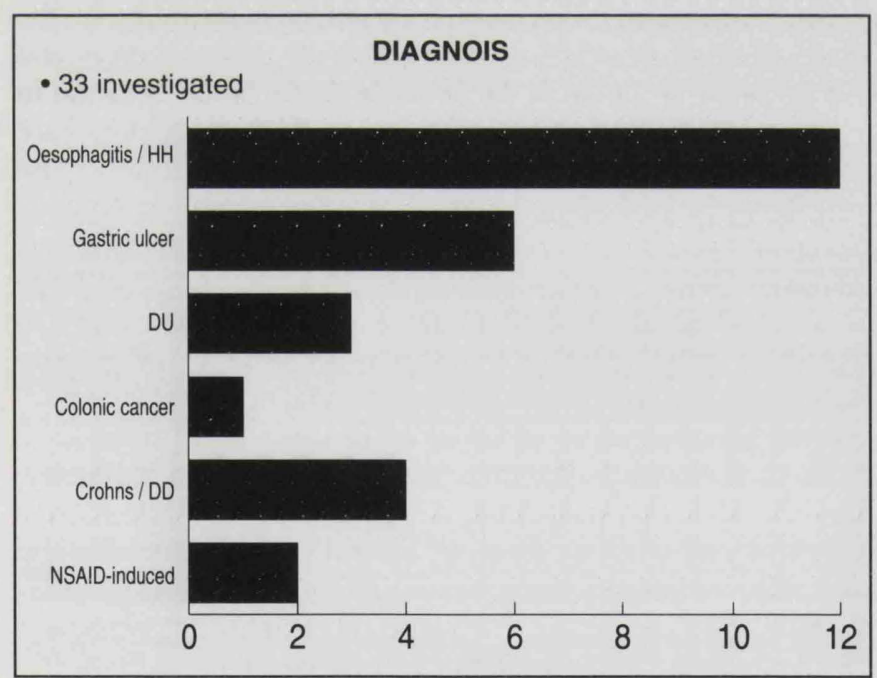

Figure 7 Diagnosed causes of iron deficiency anaemia

Since the cohort was selected during 1994 and 1995, there was an opportunity to examine longterm outcome and any major liability associated with the significant under- investigation of patients with IDA. Eleven patients subsequently developed recurrence of anaemia. One patient who was previously uninvestigated, returned as an emergency with perforation of a duodenal ulcer. This was potentially preventable. Two patients were later fully investigated with no cause found and one patient was subsequently found to have an "anaemia of chronic disorder", defined on a marrow examination.

\section{SUMMARY}

This audit has demonstrated a very significant degree of underinvestigation of those patients with iron deficiency anaemia within the department of medicine. This problem is probably also present in other hospital departments and in primary care. Apart from one later presentation with a perforated peptic ulcer there were no other late presentations with serious disease. We should not, however, take this as comforting since the sample size is small and previously published audits have demonstrated that the single most common serious missed diagnosis is colon cancer. We would therefore re-affirm our advice to colleagues, that 66 all males and all females over 50 years with iron deficiency anaemia should be investigated for an underlying cause unless there is a clear clinical reason not to. 99 all males and all females over 50 years with iron deficiency anaemia should be investigated for an underlying cause unless there is a clear clinical reason not to.

\section{Acknowledgements}

The authors wish to thank Bob James for generating the audit database of patients from pathology database, Yvonne Dickinson (Medical Illustration) and Sue Newall for producing the figures. We also wish to thank Kathleen Knipe and colleagues from the Clinical Audit Department for all their expert support in this, and many other aspects of clinical audit, and Alison Harry and Mike Flanagan for their editorial patience.

\section{REFERENCES}

1 Rockey DC, Cello CP. Evaluation of the gastrointestinal tract in patients with iron deficiency anaemia $\mathrm{N}$ Engl J Med 1993;329:1691-5

2 McIntyre AS, Long RG. Prospective survey of investigations in outpatients referred with iron deficiency anaemia and faecal occult blood loss Gut 1992;34:1102-7

3 Lucas CA, Logan ECM, Logan RFA. Audit of the investigation and outcome of irondeficiency anaemia in one health district. J Roy Coll Phys of London 1996,30:33-6 